\title{
Effects of surface tension on the size-dependent ferroelectric characteristics of free-standing $\mathrm{BaTiO}_{3}$ nano-thin films
}

\author{
Yu Su, ${ }^{1}$ Haitao Chen, ${ }^{2}$ Jacqueline J. Li, ${ }^{3}$ Ai Kah Soh, ${ }^{2}$ and George J. Weng ${ }^{4, a)}$ \\ ${ }^{1}$ Department of Mechanics, School of Aerospace Engineering, Beijing Institute of Technology, \\ Beijing, 100081, People's Republic of China \\ ${ }^{2}$ Department of Mechanical Engineering, The University of Hong Kong, Hong Kong, China \\ ${ }^{3}$ Department of Mechanical Engineering, The City College of New York, New York 10031, USA \\ ${ }^{4}$ Department of Mechanical and Aerospace Engineering, Rutgers University, New Brunswick, New Jersey \\ 08903, USA
}

(Received 2 September 2011; accepted 10 September 2011; published online 20 October 2011)

\begin{abstract}
Intrinsic surface tension of nanoscale ferroelectric thin film tends to induce tensile stress in its surface layer, whereas the other portion of the film is subjected to compression to maintain mechanical balance. A continuum-based phase-field model accounting for such surface effect has been set up to investigate the evolution of domain structure and thickness-dependent ferroelectric properties of free-standing $\mathrm{BaTiO}_{3}$ nano-thin films. It was observed that both remnant polarization and coercive field decrease with a decrease of film thickness and increase of surface tension, and that, for film thickness ranging from $10-20 \mathrm{~nm}$, both properties decreased sharply at the surface strain $2-3 \varepsilon_{0}\left(\varepsilon_{0}\right.$ being the spontaneous strain). Further decrease in film thickness or increase in surface tension could result in loss of ferroelectricity. Such a critical state for the ferroelectric-to-paraelectric transition has also been established for the range of film thickness 4-20 nm. ㄷ 2011 American Institute of Physics. [doi:10.1063/1.3652906]
\end{abstract}

\section{INTRODUCTION}

Thickness dependence of ferroelectric properties for nanoscale ferroelectric thin films has been widely reported in recent literatures. ${ }^{1-7}$ Such size dependence is generally considered as a result of several combined factors such as significantly enlarged depolarization field, ${ }^{8,9}$ interface effects, ${ }^{10,11}$ and lattice mismatch strain. ${ }^{12}$ In addition, it has been suggested that intrinsic surface stress along the free surfaces of nanostructures may also cause considerable size dependence of the properties such as the stability of ferroelectricity, change of transition temperature, polarization distribution, dielectric constants, and surface dissipation. ${ }^{13-17}$

It is well known that nanoscale materials possess higher energy at the free surfaces leading to surface tension. ${ }^{18-22}$ The material under the free surfaces must sustain an equal amount of resultant forces to maintain the mechanical balance. Although it remains a challenge to fabricate highquality free-standing ferroelectric nano-thin films, it must be reminded that strictly two-dimensional, one-atomic-thickgraphene layer has already been demonstrated to exist, and even possesses exceptional mechanical and electrical properties. ${ }^{23}$ Study of a free-standing nano-thin film allows us to isolate the effect of surface tension from other factors and it could bring new insights into its size-dependent properties.

In this article, we will apply a thermodynamics-based continuum phase field model to investigate the effect of surface tension on the domain structure and ferroelectric properties of free-standing $\mathrm{BaTiO}_{3}$ films.

\footnotetext{
a) Author to whom correspondence should be addressed. Electronic mail: weng@jove.rutgers.edu.
}

\section{THE THERMODYNAMICS-BASED CONTINUUM PHASE FIELD MODEL}

In recent years, there have been increasing activities in phase field simulations of microstructure evolution. ${ }^{24}$ This approach has also been used to study the domain structures $^{25-27}$ and ferroelectric properties ${ }^{28,29}$ of thin films. Domain structures in ferroelectric islands, overall properties of ferroelectric polycrystals, and vortex structure in single crystal nanodots, have also been investigated..$^{30-33}$ Despite the extensive phase field simulations, the influence of surface tension on the size-dependent ferroelectric characteristics of free-standing $\mathrm{BaTiO}_{3}$ nano-thin films has never been investigated. To this end, a phase-field model based on the framework of Ref. 34 is created.

To briefly recapitulate, the Helmholtz free energy of the system in this model is set to depend on strain, electric displacement, an order parameter, and its gradient, i.e., $\psi\left(\varepsilon_{i j}, D_{i}, P_{i}, P_{i, j}\right)$, where $\varepsilon_{i j}(i, j=1,2,3)$ is the components of strain tensor and $D_{i}$ the components of electric displacement vector. The spontaneous polarization $P_{i}$ is taken to be the order parameter and the subscript " $\mathrm{j}$ " represents partial differentiation with respect to the $\mathrm{x}_{\mathrm{j}}$ coordinate direction. The work-conjugates of these parameters are stress, electric field, and micro-forces, respectively. Within the theory of linear piezoelectricity, the mechanical and electrical quantities of above-mentioned terms should satisfy the fundamental balance laws, i.e., balances of linear and angular momentum, the quasi-static forms of Maxwell's equations, balances of micro-forces, and the kinematic relationships. Under a thermodynamically consistent framework, one can derive the constitutive relationships that relate the parameters of the free energy function to their work-conjugates by analyzing the second law of thermodynamics, and consequently obtain 
the governing equation that describes the temporal evolution of the spontaneous order parameters as

$$
\left(\frac{\partial \psi}{\partial P_{i, j}}\right)_{, j}-\frac{\partial \psi}{\partial P_{i}}+\gamma_{i}=\beta_{i j}\left(\frac{\partial P_{j}}{\partial t}\right) .
$$

Equation (1) is essentially the generalized form of GinzburgLandau equation, where $\gamma_{i}$ represents the components of external micro-force vector, $\beta_{i j}$ the components of inverse mobility tensor, and $t$ the time. Standard index notation is used with summation implied over repeated indices throughout this article. A general form of the free energy $\psi\left(\varepsilon_{i j}, D_{i}, P_{i}, P_{i, j}\right)$ can be given as

$$
\begin{aligned}
\psi= & \frac{1}{2} a_{i j k l} P_{i, j} P_{k, l}+\left\{\frac{1}{2} \bar{a}_{i j} P_{i} P_{j}+\frac{1}{4} \overline{\bar{a}}_{i j k l} P_{i} P_{j} P_{k} P_{l}\right. \\
& \left.+\frac{1}{6} \overline{\bar{a}}_{i j k l m n} P_{i} P_{j} P_{k} P_{l} P_{m} P_{n}+\frac{1}{6} \overline{\overline{\bar{a}}}_{i j k l m n r s} P_{i} P_{j} P_{k} P_{l} P_{m} P_{n} P_{r} P_{s}\right\} \\
& +\left\{b_{i j k l} \varepsilon_{i j} P_{k} P_{l}+\frac{1}{2} c_{i j k l} \varepsilon_{i j} \varepsilon_{k l}+f_{i j k l m n} \varepsilon_{i j} \varepsilon_{k l} P_{m} P_{n}\right. \\
& \left.+g_{i j k l m n} \varepsilon_{i j} P_{k} P_{l} P_{m} P_{n}\right\}+\frac{1}{2 \kappa_{0}}\left(D_{i}-P_{i}\right)\left(D_{i}-P_{i}\right),
\end{aligned}
$$

where $\kappa_{0}$ is the permittivity of free space. The first term of the free energy penalizes large gradients of polarization. The four terms in the first brace are used to create the nonconvex energy landscape of the free energy with minima located at the spontaneous polarization states. The four terms in the second brace are used to fit the spontaneous strain along with the dielectric, elastic, and piezoelectric properties about the spontaneous state. Note that the elastic, dielectric, and piezoelectric properties are non-linear, therefore, the tensor components are fit to the tangent material properties at the spontaneously polarized state. The final term represents the energy stored within the free space occupied by the material. This model is good for isothermal process below the $\mathrm{Cu}$ rie temperature and needs to be extended if temperature dependence is considered. In this work, a principle of virtual work is specified for the theory and is implemented to devise a finite element formulation. One can then investigate the effects of surface tension on ferroelectric nano-thin films with such phase field approach.

\section{THE SURFACE TENSION AND BOUNDARY CONDITION IN THE MODEL}

A free standing thin film can be treated as a twodimensional (2D) problem with free boundaries on both top and bottom surfaces subjected to tensile stresses, as shown in Fig. 1(a). Since the geometry is symmetric, the problem can be further simplified by modeling only half of the film thickness, as shown in Fig. 1(b), with appropriate boundary conditions implemented as follows. For boundaries on the two sides $\left(\mathrm{x}_{1}=0\right.$ and $\left.\mathrm{x}_{1}=l\right)$, the in-plane displacement $\mathrm{u}_{1}$ is fixed along $\mathrm{x}_{1}=0$ and is set free along $\mathrm{x}_{1}=l$; the electric potential and polarization on both sides are set to be identical to satisfy the electric periodic boundary condition. For the boundary at the bottom $\left(\mathrm{x}_{3}=0\right)$, the out-of-plane displacement $\mathrm{u}_{3}$ and electric potential are fixed to zero according to symmetry of geometry; free polarization boundary condition is assumed at the bottom as $\partial P_{i} / \partial n_{j}=0$, which implies infinite extrapolation length. The boundary on the top $\left(x_{3}=h / 2\right)$ is set free except for a uniformly prescribed electric potential $\phi$, through which the controlled overall electric field can be applied to the thin film along the thickness direction. To consider the effect of surface stress, in-plane tensile strain, $\varepsilon_{11}^{s}$, is prescribed to the very top region of the nano-thin film with thickness $h_{s}$.

The considered ferroelectric material is $\mathrm{BaTiO}_{3}$ and the material properties are adopted from Ref. 34. The simulation is conducted on the geometric model shown in Fig. 1(b) which has been meshed for finite element computing. The minimum grid spacing is $0.5 \mathrm{~nm}$. The length $l$ of the periodic unit is set to be $800 \mathrm{~nm}$ and the total thickness $h$ of the film is subject to change from several to tens of nanometers. The thickness of the pre-tensioned top region is fixed at $h_{s}=1 \mathrm{~nm}$, regardless of the film thickness. The whole computing procedure is assumed to be an isothermal process below the Curie temperature. The computation starts with an initial condition at which the spontaneous polarizations form $180^{\circ}$ stripe domain structures. ${ }^{6,35}$ The total number of time

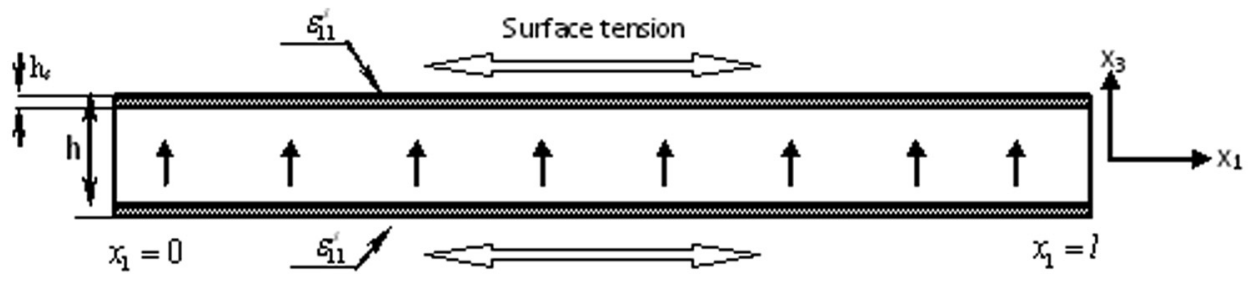

(a)

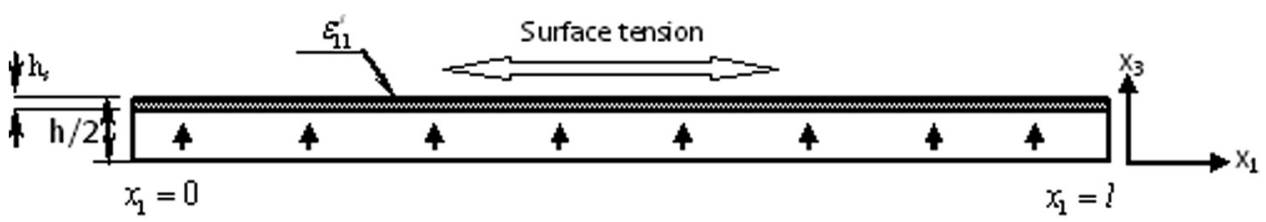

(b)
FIG. 1. The geometric model for a freestanding $\mathrm{BaTiO}_{3}$ nano-thin film: (a) the full model and (b) the half-thickness model. 


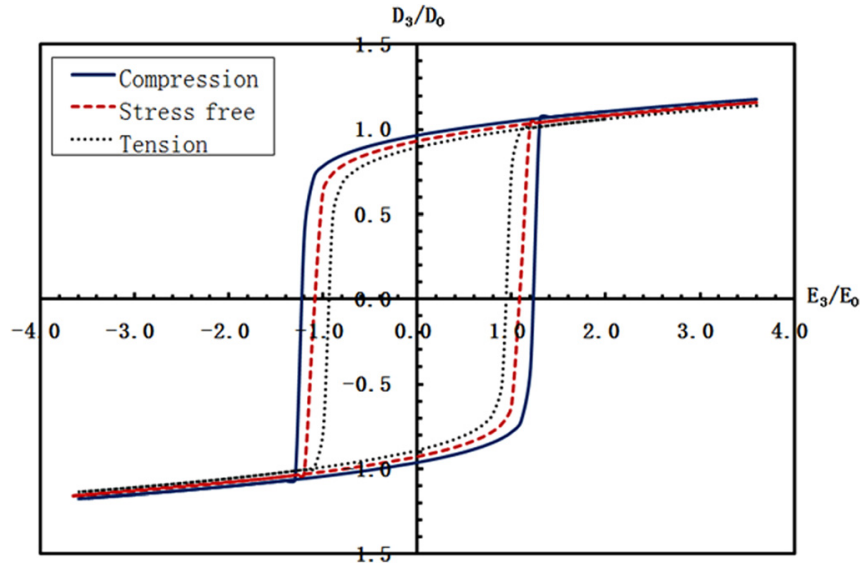

FIG. 2. (Color online) The hysteresis loops of $80 \mathrm{~nm}$-thick free-standing $\mathrm{BaTiO}_{3}$ nano-thin film with different uniform loading conditions over the whole thickness without surface stress. The characteristic electric displacement and electric field are $\mathrm{D}_{0}=0.26 \mathrm{C} / \mathrm{m}^{2}$ and $\mathrm{E}_{0}=21.8 \mathrm{MV} / \mathrm{m}$.

steps for each increment of electric field is set to be large enough so that the system can reach full equilibrium state.

Before we proceed to the results with surface tension, it is instructive to see the results calculated by this model without surface stress first. In this case, uniform in-plane volume compression and volume tension are applied separately over the entire thickness of the nano-thin film $(\mathrm{h}=80 \mathrm{~nm})$. The calculated hysteresis loops-along with that of the stressfree state-are given in Fig. 2, where the reference electric displacement $D_{0}$ and electric field are $D_{0}=0.26 \mathrm{C} / \mathrm{m}^{2}$ and $\mathrm{E}_{0}=21.8 \mathrm{MV} / \mathrm{m}$, respectively. It is seen that the film has greater remnant polarization and coercive field under compression and lower values under tension. The calculated trend is consistent with what have been reported in Refs. 28 and 29 for epitaxial thin films under uniform lateral tension/ compression, and it also serves to validate this phase-field model.

\section{EFFECTS OF SURFACE TENSION}

We now present the effect of surface tension. The first set of computational results is shown in Fig. 3, with the film thickness of $20 \mathrm{~nm}$ and $10 \mathrm{~nm}$, and surface tensile strain of $\varepsilon_{11}^{s}=0,0.5 \varepsilon_{0}$, and $\varepsilon_{0}$, where $\varepsilon_{0}=0.82 \%$ is the spontaneous strain along the c-axis. It is evident that, within this range of surface tension, both remnant polarization and coercive field tend to decrease with decreasing film thickness and increasing surface tension.

To give a detailed picture on how the polarizations redistribute during the hysteresis loop computation, we present the evolution of domain structure as the electric field changes for the $20 \mathrm{~nm}$-thick film with prescribed strain of $0.5 \varepsilon_{0}$. The results are given in Figs. 4(a)-4(e), which correspond to positions A, B, C, D, and E, respectively, in Fig. 3. Fig. 4(a) is the initial $180^{\circ}$ stripe domain. As the field increases some of the downward polarization starts to switch up, as shown in Fig. 4(b). Fig. 4(c) represents the fully polarized state. Fig. 4(d) gives the domain pattern as the field is approaching the coercive value, and Fig. 4(e) shows the domain state after the electric field passed the coercive field. The obtained $180^{\circ}$

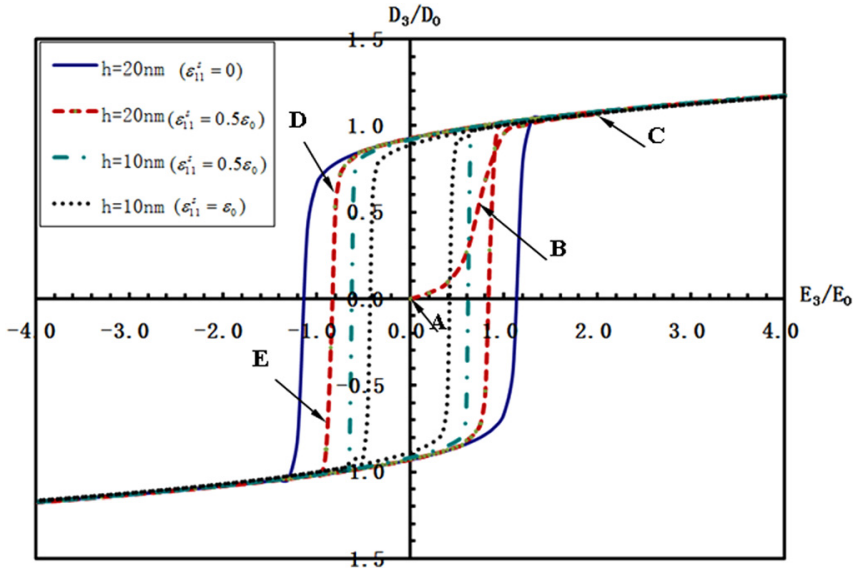

FIG. 3. (Color online) Hysteresis loops of free-standing $\mathrm{BaTiO}_{3}$ nano-thin films with different film thickness and prescribed surface strains.

stripe domain is very similar to the results obtained by the first principles calculation for lead zirconate titanate $(\mathrm{PZT}),{ }^{36}$ and now it is demonstrated that $\mathrm{BaTiO}_{3}$ can have similar domain structure. In all these figures, a distinct domain layer near the surface is visible due to the action of surface tension.

To uncover the effect of surface tension over a broader range of film thickness, we further conducted simulations on $\mathrm{BaTiO}_{3}$ nano-thin films with thickness ranging from $40 \mathrm{~nm}$ down to $4 \mathrm{~nm}$. The computation was run with the prescribed

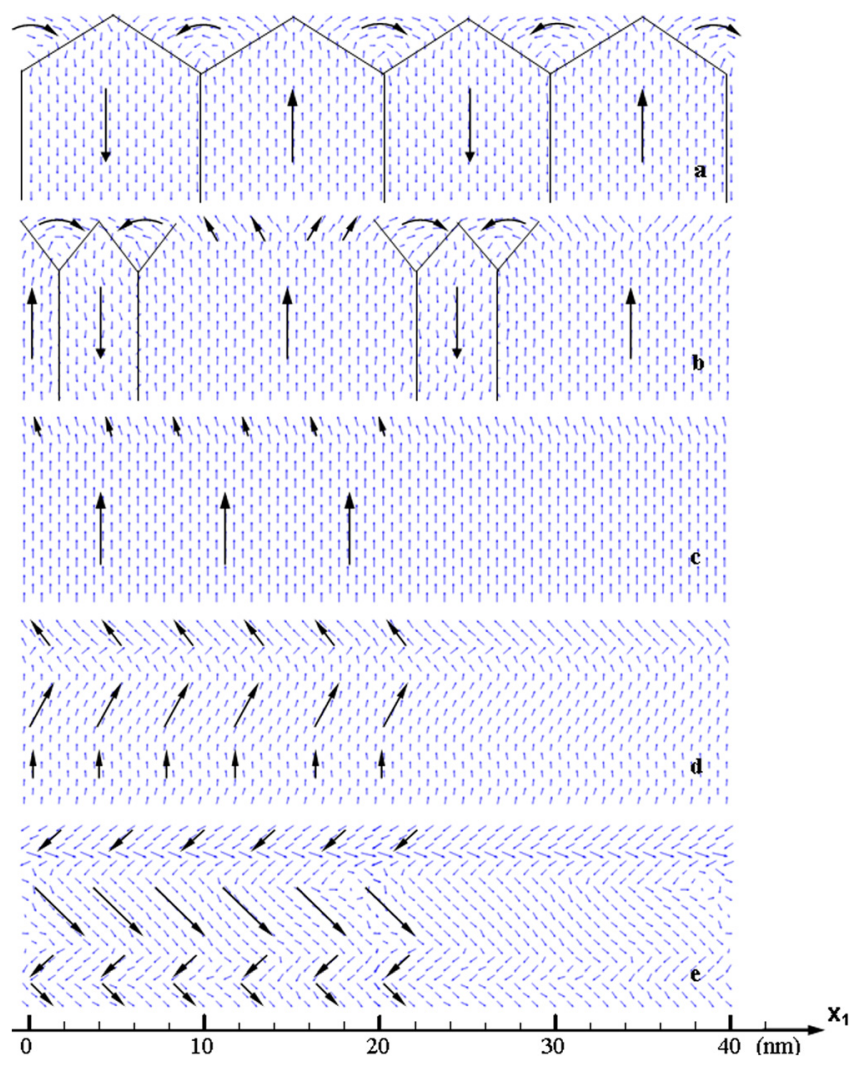

FIG. 4. (Color online) The distributions of polarization for the $20 \mathrm{~nm}$ thin film at selected states as marked in Fig. 3: (a) the $180^{\circ}$ stripe domain configuration at the initial state; (b) the subsequent state with upward applied electric field $\mathrm{E}_{3}=0.7 \mathrm{E}_{0}$; (c) the completely poled state with upward electric field $\mathrm{E}_{3}=2.0 \quad \mathrm{E}_{0}$; (d) the state with reversely applied electric field $\mathrm{E}_{3}=-0.8 \mathrm{E}_{0}$; and (e) the switched state at $\mathrm{E}_{3}=-0.84 \mathrm{E}_{0}$. 


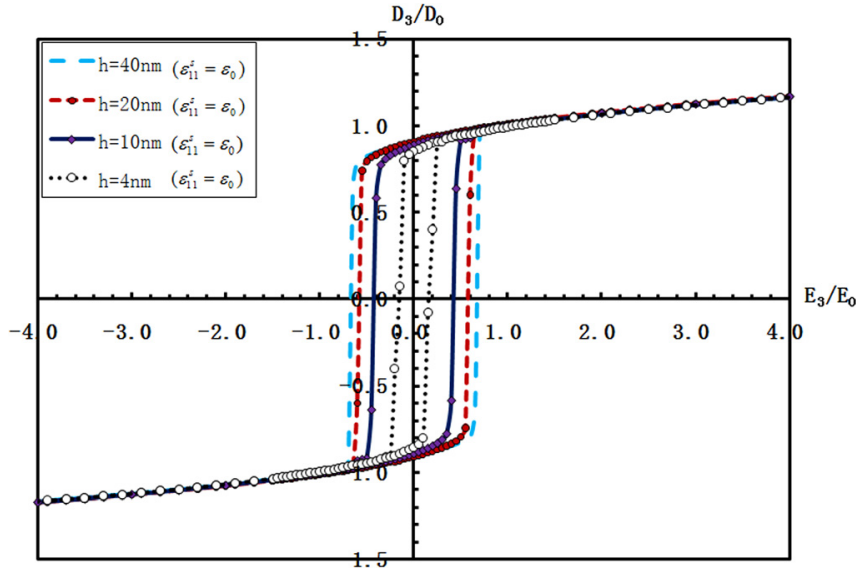

FIG. 5. (Color online) The hysteresis loop for different film thickness with prescribed surface strain of $\varepsilon_{0}$.

surface strain of $\varepsilon_{0}$ and $2 \varepsilon_{0}$, and the obtained hysteresis loops are illustrated in Figs. 5 and 6, respectively $\left(\mathrm{P}_{0}=0.26 \mathrm{C} / \mathrm{m}^{2}\right.$ and $\mathrm{E}_{0}=21.8 \mathrm{MV} / \mathrm{m}$ ). It is clear from Fig. 5 that both remnant polarization and coercive field decrease with a decrease in film thickness. At the higher surface strain shown in Fig. 6, the loops with the film thickness of $10 \mathrm{~nm}$ and $4 \mathrm{~nm}$ have become very slanted. This trend points to the possibility that a free-standing ferroelectric nano-thin film could lose its ferroelectricity if the film thickness is low and its surface tension is high.

We have made additional calculations for the variation of remnant polarization and coercive field with increasing levels of surface strain, for the film thickness 20 and $10 \mathrm{~nm}$. The obtained results are shown in Fig. 7. It is found that initially, the remnant polarization decreases moderately but then drops sharply at $2-3 \varepsilon_{0}$. The coercive field is found to drop sharply from $0 \varepsilon_{0}$ down to $2-3 \varepsilon_{0}$, and then increase and decrease again. The sharp drop of the remnant polarization shown here is in direct contrast to the gradual decrease reported for epitaxial films in Ref. 28, but the decrease and then increase of the coercive field is very similar to what has been reported there.

We have also made additional calculations over a wide range of film thickness from $100 \mathrm{~nm}$ down to $4 \mathrm{~nm}$, with the

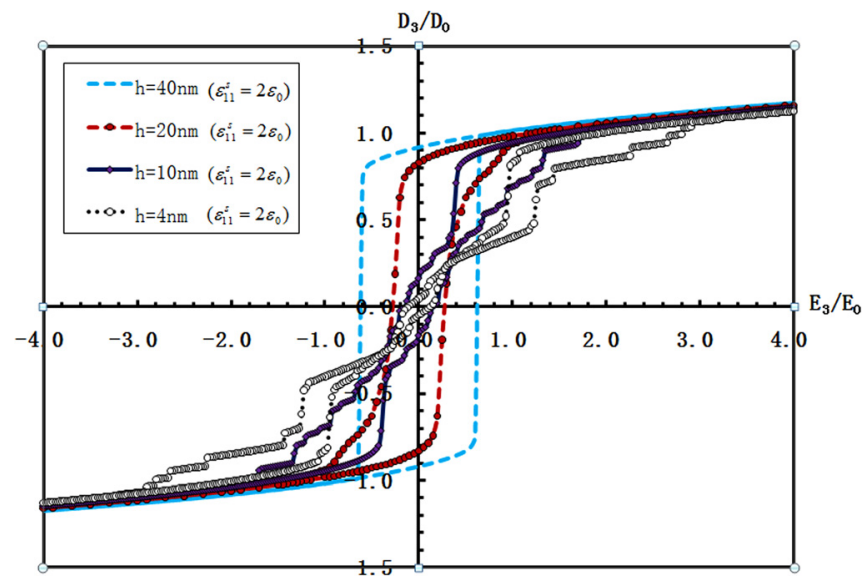

FIG. 6. (Color online) The hysteresis loop for different film thickness with prescribed surface strain of $2 \varepsilon_{0}$.

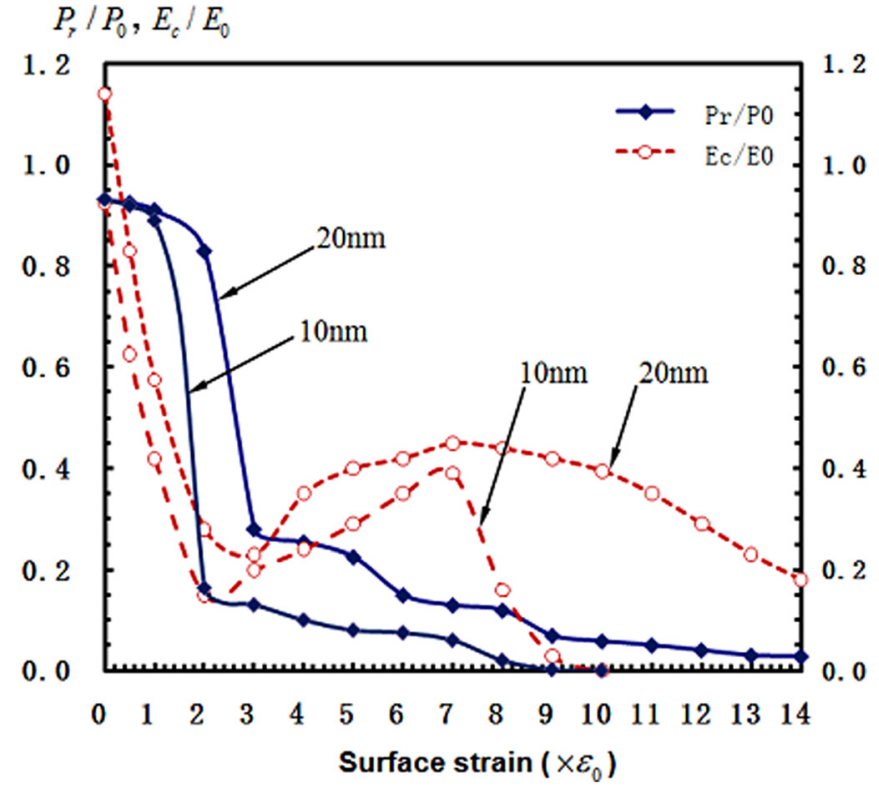

FIG. 7. (Color online) The coercive electric field and remnant polarization as a function of surface strain.

surface strains, $\varepsilon_{0}$ and $2 \varepsilon_{0}$. The results for both remnant polarization and coercive field are shown in Fig. 8. Both quantities are seen to remain fairly constant until the film thickness has decreased to about $20 \mathrm{~nm}$ for the remnant polarization, and to about $30 \mathrm{~nm}$ for the coercive field. After that the decrease becomes apparent.

A closer look at Fig. 7 for the case of $10 \mathrm{~nm}$ film under the surface strain $9 \varepsilon_{0}$ and at Fig. 8 for the case of $2 \varepsilon_{0}$ tension for the film thickness $4 \mathrm{~nm}$ reflects a diminishing value of $\mathrm{P}_{\mathrm{r}}$ and $\mathrm{E}_{\mathrm{c}}$. That is, the film is on the verge of approaching to the state of ferroelectric to paraelectric transition. Moreover, it also suggests that for a film with a larger thickness $(10 \mathrm{~nm}$ here), the required surface strain is higher $\left(9 \varepsilon_{0}\right)$, and for a thinner film $(4 \mathrm{~nm})$, the required surface tension is lower

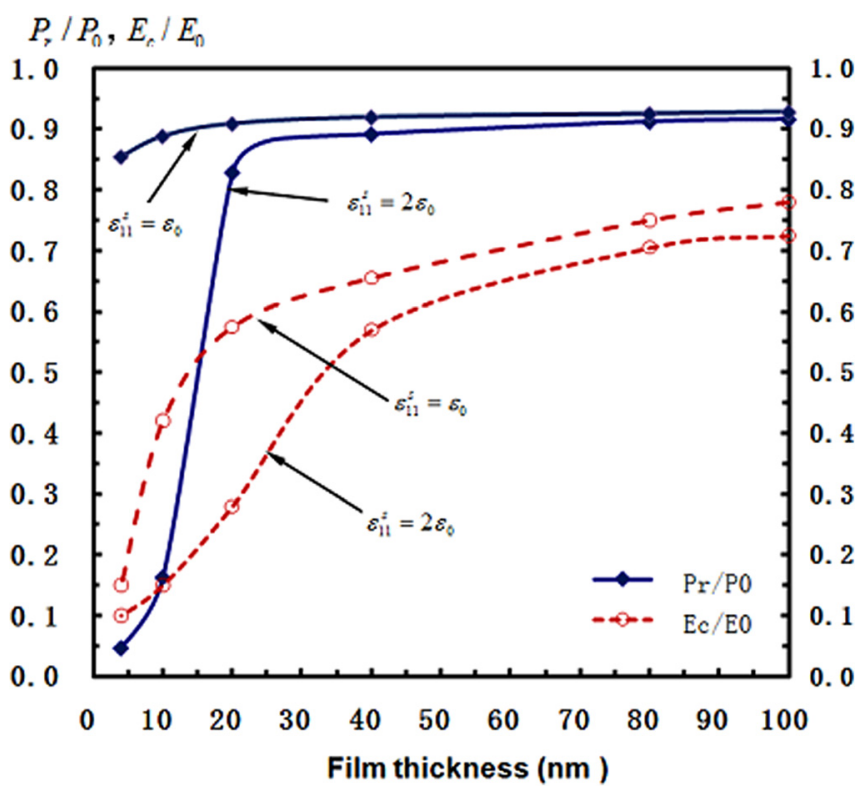

FIG. 8. (Color online) The coercive electric field and remnant polarization as a function of film thickness. 


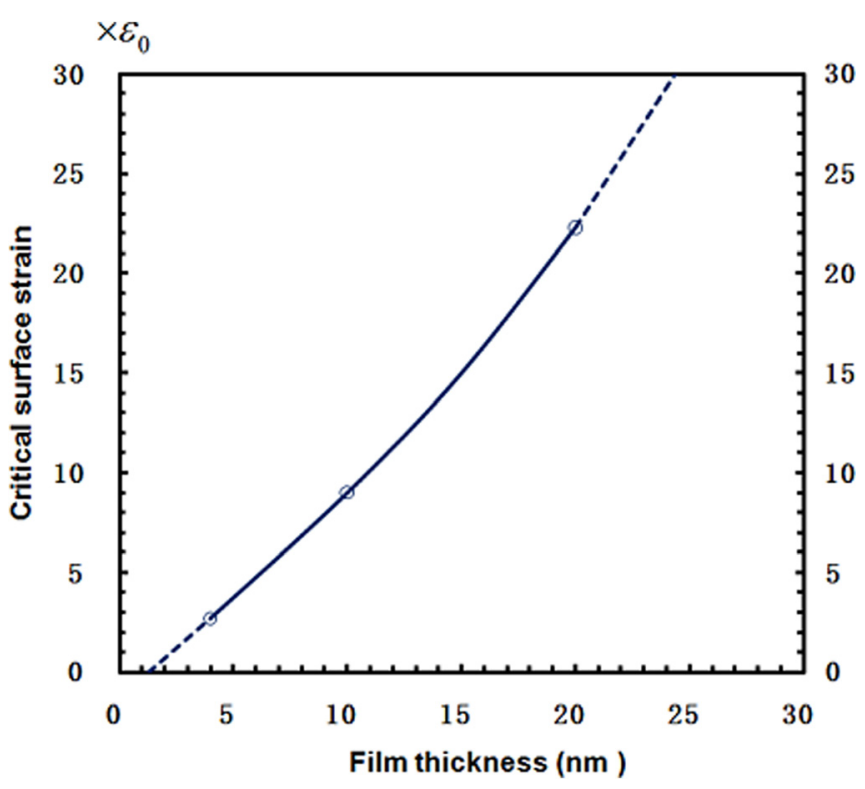

FIG. 9. (Color online) The critical surface strain as a function of film thickness to turn ferroelectric film into paraelectric.

$\left(2 \varepsilon_{0}\right)$ for the paraelectric state to be reached. These limiting cases imply that, in spite of the fact that surface tension acts only over a very thin surface layer, it is capable of causing a free-standing film to lose its ferroelectricity. We decided to conduct a more comprehensive study on this issue by calculating the required critical surface strain for a given film thickness within the range 4-20 nm. The results are shown in Fig. 9. A monotonic increase of surface tension is required, and the relation, as can be expected, shows a convex nature between these two parameters.

\section{ANALYSIS OF THE THICKNESS DEPENDENCE OF REMNANT POLARIZATION AND COERCIVE FIELD}

To make a simple explanation for the nonlinear thickness-dependent trend of the film shown in Fig. 8, let us denote the physical quantities in the surface-tensioned region with a superscript (1) and the portion below it with superscript (2) (see Fig. 4(c), for instance). The quantities in a free standing reference nano-thin film without surface stress are denoted with superscript (0). In the current phase field model, the electric field, electric displacement, and polarization must satisfy the relation $D_{i}=\kappa_{0} E_{i}+P_{i} \quad(I=1,2,3)$, where $\kappa_{0}$ is the dielectric permittivity of free space. We now assume for simplicity that polarizations in both regions are homogenous (could be conceived as the average of the heterogeneous phase-field results), and note that, under an applied $E_{3}$, the electric displacement is constant along the thickness direction, such that

$$
\begin{aligned}
& D_{3}^{(1)}=\kappa_{0} E_{3}^{(1)}+P_{3}^{(1)}, \quad D_{3}^{(2)}=\kappa_{0} E_{3}^{(2)}+P_{3}^{(2)}, \quad \text { and } \\
& D_{3}^{(1)}=D_{3}^{(2)}=D_{3}
\end{aligned}
$$

Given that the total electric displacement $D_{3}$ vanishes at the coercive electric field $E_{c}$, Eq. (3) yields $E_{c}^{(1)}=-P_{c}^{(1)} / \kappa_{0}$ and $E_{c}^{(2)}=-P_{c}^{(2)} / \kappa_{0}$, where $P_{c}^{(1)}$ and $P_{c}^{(2)}$ are the average polarizations of regions 1 and 2 , respectively. The overall coer- cive field of the film is given by the weighted mean of the two as

$$
E_{c}=f_{1}\left\langle E_{c}^{(1)}\right\rangle+f_{2}\left\langle E_{c}^{(2)}\right\rangle=-\left(f_{1}\left\langle P_{c}^{(1)}\right\rangle+f_{2}\left\langle P_{c}^{(2)}\right\rangle\right) / \kappa_{0},
$$

where $f_{1}$ and $f_{2}$ are the volume fractions occupied by regions (1) and (2), respectively, with $f_{1}+f_{2}=1$. The angle brackets are added here to emphasize that they stand for the volumeaveraged quantity. For the reference thin film without surface stress, one has

$$
E_{c}^{(0)}=-\left\langle P_{c}^{(0)}\right\rangle / \kappa_{0}
$$

Taking the volume average of our phase-field data, we found that $\left|\left\langle P_{c}^{(1)}\right\rangle\right|\left\langle\left|\left\langle P_{c}^{(2)}\right\rangle\right| \leq\left|\left\langle P_{c}^{(0)}\right\rangle\right|\right.$ at the coercive state. With the additional factor that $f_{1}$ is finite and increases with decreasing film thickness, we can see that $E_{c}<E_{c}^{(0)}$. Likewise, the overall remnant polarization at zero total applied electric field $E_{3}$ is given by

$$
P_{r}=\left(f_{1}\left\langle P_{3}^{(1)}\right\rangle+f_{2}\left\langle P_{3}^{(2)}\right\rangle\right) \leq P_{r}^{(0)},
$$

where $P_{r}^{(0)}$ is the remnant polarization of the reference film without surface stress. Eq. (3) through Eq. (6) mathematically explain why, under the action of surface tension, both coercive field and remnant polarization decrease with a decrease in film thickness. It should also be emphasized that, although Eqs. (4) and (6) represent linear dependence of coercive field and remnant polarization on the volume fractions of separate film regions, the dependence of polarization on the film thickness in each phase is nonlinear. To illustrate this point more clearly, we have plotted the averaged polarizations $\left\langle P_{3}^{(1)}\right\rangle$ and $\left\langle P_{3}^{(2)}\right\rangle$ of both phases (1) and (2) with the surface tensile strain of $2 \varepsilon_{0}$ in Fig. 10, at zero field. It can be seen that both decrease drastically as the film thickness

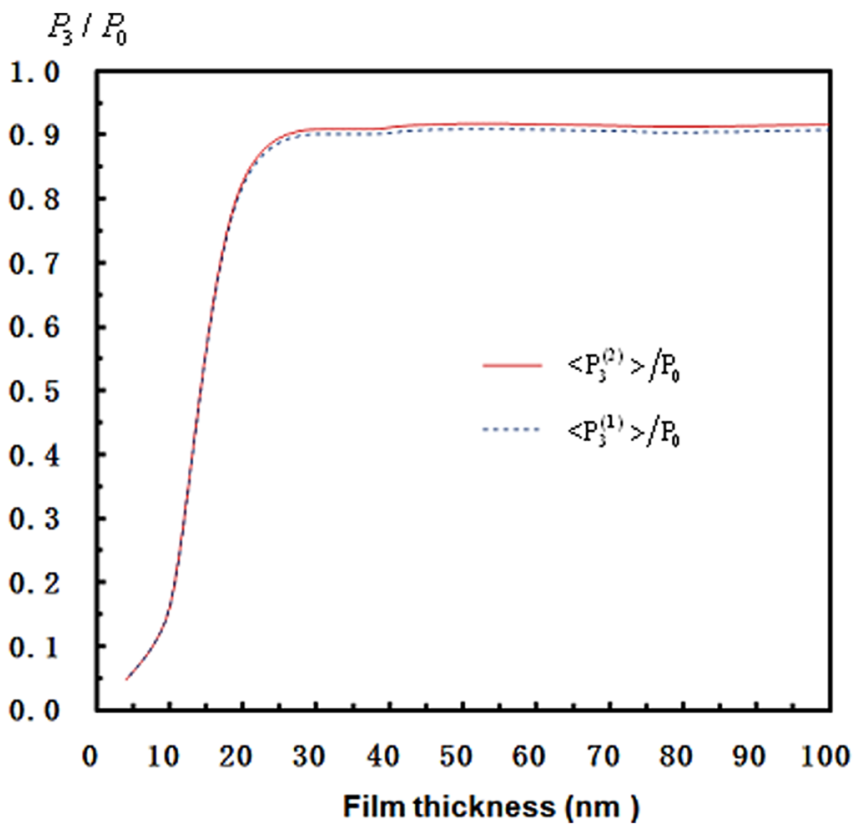

FIG. 10. (Color online) The averaged polarizations in the top surface region (region 1) and interior (region 2) of the nano-thin film as a function of film thickness. 
decreases to $20 \mathrm{~nm}$. This drastic decrease-following Eq. (6) - translates into drastic decrease of $P_{r}$ for the overall film, reflected in Fig. 8.

\section{CONCLUSIONS}

Phase field approach has been extensively used to study the domain structure and ferroelectric properties of thin films, but it has never been used to examine the influence of surface tension on the ferroelectric characteristics of a freestanding nano-thin films. In this article, we have applied a thermodynamics-based continuum phase field model to examine its influence on the evolution of domain structure and change of remnant polarization and coercive field over the range of film thickness from $40 \mathrm{~nm}$ down to $4 \mathrm{~nm}$. We first verified its validity by simulating thin films with a uniform lateral strain across the entire thickness that is similar to the epitaxial strain studied by others. We then applied it to study the effect of surface tension on the remnant polarization and coercive field of the film. It is demonstrated that, even though the surface tension spans over only a thin layer, its influence on the ferroelectric characteristics of a freestanding film can be quite significant. For a film with thickness $10-20 \mathrm{~nm}$, both remnant polarization and coercive field are found to decrease sharply as the surface tension increases from $1 \varepsilon_{0}$ to $3 \varepsilon_{0}$, and for a film under the action of surface tension $2 \varepsilon_{0}$, both quantities are found to decrease significantly as the film thickness decreases to $20-30 \mathrm{~nm}$. Finally, it is further demonstrated that a free-standing film could lose its ferroelectric characteristics if the film thickness is too low or the surface tension is too high. The critical state that gives rise to such ferroelectric-to-paraelectric transition is established for the range of film thickness 4-20 nm.

\section{ACKNOWLEDGMENTS}

Y. Su thanks the support of National Natural Science Foundation of China under the Grant No. 11002024 and 11132011. J. Li was supported by NSF-CMMI-IDR1014777, and A. K. Soh by RGC of Hong Kong SAR (Project Nos. HKU716007E and 716508E) Both $\mathrm{Li}$ and Soh would also like to thank the William M.W. Mong Engineering Research Fund from HKU. G. J. Weng thanks the support of NSF CMS 0510409 and the University of Hong Kong Visiting Research Professor Scheme 2010-2013.
${ }^{1}$ S. Rios, J. F. Scott, A. Lookman, J. McAneney, R. M. Bowman, and J. M. Gregg, J. Appl. Phys. 99, 024107 (2006).

${ }^{2}$ Y. Wang, Y. H. Lin, and C. W. Nan, J. Appl. Phys. 104, 123912 (2008).

${ }^{3}$ Y. Bastani, T. Schmitz-Kempen, A. Roelofs, and N. Bassiri-Gharb, J. Appl. Phys. 109, 014115 (2011).

${ }^{4}$ J. Wang and T. Y. Zhang, Phys. Rev. B 73, 144107 (2006).

${ }^{5}$ Y. L. Sang, B. Liu, D. N. Fang, Comput. Mater. Sci. 44, 404 (2008).

${ }^{6}$ L. Hong, A. K. Soh, Y. C. Song, and L. C. Lim, Acta Mater. 56, 2966 (2008).

${ }^{7}$ Y. Y. Liu and J. Y. Li, Appl. Phys. Lett. 97, 042905 (2010).

${ }^{8}$ R. R. Mehta, B. D. Silverman, and J. T. Jacobs, J. Appl. Phys. 44, 3379 (1973).

${ }^{9}$ D. J. Kim, J. Y. Jo, Y. S. Kim, Y. J. Chang, J. S. Lee, J. G. Yoon, T. K. Song, and T. W. Noh, Phys. Rev. Lett. 95, 237602 (2005).

${ }^{10}$ J. Q. He, E. Vasco, C. L. Jia, and R. H. Wang, Appl. Phys. Lett. 87, 062901 (2005).

${ }^{11}$ Y. Park and C. S. Hwang, Appl. Phys. Lett. 85, 5313 (2004).

${ }^{12} \mathrm{H}$. Li, A. L. Roytburd, S. P. Alpay, T. D. Tran, L. Salamanca-Riba, and R. Ramesh, Appl. Phys. Lett. 78, 2354 (2001).

${ }^{13}$ A. N. Morozovska, M. D. Glinchuk, and E. A. Eliseev, Phys. Rev. B 76, 014102 (2007).

${ }^{14}$ Z. H. Zhou, X. S. Gao, J. Wang, K. Fujihara, S. Ramakrishna, and V. Nagarajan, Appl. Phys. Lett. 90, 052902 (2007).

${ }^{15}$ Y. Su and J. N. Du, Appl. Phys. Lett. 96, 162905 (2010).

${ }^{16}$ C. Q. Ru, Appl. Phys. Lett. 94, 051905 (2009).

${ }^{17}$ S. M. Hasheminejad and B. Gheshlaghi, Appl. Phys. Lett. 97, 253103 (2010).

${ }^{18}$ R. Shuttleworth, Proc. Phys. Soc., London, Sect. A 63, 444 (1950).

${ }^{19}$ M. E. Gurtin and A. I. Murdoch, Arch. Ration. Mech. Anal. 57, 291 (1975).

${ }^{20}$ M. E. Gurtin and A. I. Murdoch, Int. J. Solids Struct. 14, 432 (1978).

${ }^{21}$ V. A. Shchukin and D. Bimberg, Rev. Mod. Phys. 71, 1125 (1999).

${ }^{22}$ T. Y. Zhang, M. Luo, and W. K. Chan, J. Appl. Phys. 103, 104308 (2008).

${ }^{23}$ A. K. Geim and K. S. Novoselov, Nature Mater. 6, 183 (2007).

${ }^{24}$ L. Q. Chen, Annu. Rev. Mater. Res. 32, 113 (2002).

${ }^{25}$ Y. L. Li, S. Y. Hu, Z. K. Liu, and L. Q. Chen, Appl. Phys. Lett. 78, 3878 (2001); Acta Mater. 50, 395 (2002).

${ }^{26}$ Y. L. Li and L. Q. Chen, Appl. Phys. Lett. 88, 072905 (2006).

${ }^{27}$ Y. C. Shu, J. H. Yen, H. Z. Chen, J. Y. Li, and L. J. Li, Appl. Phys. Lett. 92, 052909 (2009).

${ }^{28}$ S. Choudhury, Y. L. Li, L. Q. Chen, and Q. X. Jia, Appl. Phys. Lett. 92, 142907 (2008).

${ }^{29}$ Y. L. Sang, B. Liu, and D. N. Fang, Comput. Mater. Sci. 44, 404 (2008).

${ }^{30}$ J. X. Zhang, G. Sheng, and L. Q. Chen, Appl. Phys. Lett. 96, 132901 (2010).

${ }^{31}$ F. X. Li, X. L. Zhou, and A. K. Soh, Appl. Phys. Lett. 96, 152905 (2010).

${ }^{32}$ Y. Su and J. N. Du, Appl. Phys. Lett. 95, 012903 (2009).

${ }^{33}$ J. Wang, Appl. Phys. Lett. 97, 192901 (2010).

${ }^{34}$ Y. Su and C. M. Landis, J. Mech. Phys. Solids 55, 280 (2007).

${ }^{35}$ G. J. Weng and D. T. Wong, J. Mech. Phys. Solids 57, 571 (2009).

${ }^{36}$ B. K. Lai, I. Ponomareva, I. Kornev, L. Bellaiche, and G. Salamo, Appl. Phys. Lett. 91, 152909 (2007). 